\title{
Fuzzy artificial neural network for prediction and management tasks
}

\author{
Kibriyo Mukhamadieva ${ }^{1}$ \\ ${ }^{1}$ Bukhara Engineering Technological Institute, Q.Murtazaev 15, Bukhara, 200100, Uzbekistan
}

\begin{abstract}
In the work the task of prediction of parameters at construction of systems of forecasting and management is considered. Existing solutions in the use of fuzzy neural networks are studied and analyzed. The structure of a fully coupled fuzzy artificial neural network without a layer of fuzzy rules, corresponding to the "classical" multilayer perceptron, is proposed. Tested for learning time and RMS error of different structures of the proposed fuzzy artificial neural network in predicting the performance consumed by a coal company. The results allow you to select the number of neurons in the hidden layers depending on the desired accuracy of the prediction of the output parameter.
\end{abstract}

\section{Keywords}

fuzzy logic, fuzzy artificial neural network, perceptron, identity function (IF)

\section{Introduction}

Prediction issues are relevant for any human activity: weather forecasts, exchange rate forecasts, socio-economic forecasts, etc. In order to make these forecasts, there is usually a sufficient amount of data collected over many years, as well as data obtained from current observations or from experts. However, there are a number of specific tasks: forecasting the demand for resources of an enterprise, forecasting natural phenomena, etc., when information about an object, its parameters and states is incomplete, uncertain and/or poorly formalized. Such types of problems arise when building control systems of objects, the parameters of which are difficult to measure, or the relationship between them is not uniquely established. An example is the water treatment system of an industrial enterprise, when the number of indicators (concentration of dissolved salts and gases in water) reaches several dozens, they are all interrelated, and there is no unambiguous function to select the number of reagents for treatment depending on the initial chemical composition of water. Another example is the prediction of electricity demand for a coal mining enterprise, the energy consumption of which is a complex nonstationary process, which is influenced by a significant number of mining-geological, technological, industrial, climatic and other factors [1-2].

Among the currently known models and forecasting methods we can distinguish [2]: multiplicative models, dynamic linear and nonlinear models, threshold autoregressive models, Kalman filters, time series, ARMAX models, nonparametric regression models, artificial neural networks (ANN), statistical models, and hybrid models, such as fuzzy artificial neural networks (FANN).

Various kinds of regressions and models generated from them, as well as time series can be effectively used in cases where the dependence of the predicted indicator in time is continuous, has a smooth character and does not contain jumps and discontinuities. In the case of forecasting on the basis of non-periodic data series, in order to obtain an acceptable accuracy (at least in units of percent), a significant number of row members or regression coefficients have to be taken into account. Moreover, when processing non-periodic signals, both regression and time series give adequate results only within the interpolation interval. Artificial neural networks are more flexible than the aforementioned models,

\footnotetext{
III International Workshop on Modeling, Information Processing and Computing (MIP: Computing-2021), May 28, 2021, Krasnoyarsk, Russia

EMAIL: mkb78@mail.ru (K. Mukhamadieva)

ORCID: 0000-0002-4436-6333 (K. Mukhamadieva)

(c) (i) (C) 2021 Copyright for this paper by its authors.

Use permitted under Creative Commons License Attribution 4.0 International (CC BY 4.0).

CEUR Workshop Proceedings (CEUR-WS.org)
} 
which is explained by the presence of a complete relationship between the input and intermediate variables, as well as by the possibility of introducing nonlinearity into the activation functions [3]. This explains their expanding application in solving computational, statistical, prognostic and other problems. A feature of "classical" ANNs is that in order to train them it is necessary to have a sufficiently large amount of initial data, which is not always possible. To overcome the limitations inherent to "classical" ANN, fuzzy artificial neural networks were developed that use the theory of fuzzy sets, which makes it possible to build predictive models for cases of uncertainty or lack of input data. The introduction of such fuzzy is possible for input and output data, weights of neurons, as well as for the production of intermediate transformations [4].

\section{Main part}

To date, more than a dozen varieties of fuzzy artificial neural networks are known: Takagi-SugenoKang (TSK), Wang-Mendel (WM), adaptive ANFIS, FALCON, GARIC, NEFCON and FUN FANN, fuzzy multilayer perceptron, hybrid neural network, as well as FANN, which are various modifications of "classical" ANS (Kohonen fuzzy self-organizing network, fuzzy baseline radial network and others.) [4-6].

While "classical" ANNs are presented in the literature as a universal tool for processing unrelated to the subject domain data, FANN, as a rule, are focused on a narrower range of tasks. Thus, Takagi Sugheno Kanga and Wang-Mendel FANN are used for data classification and prediction [3], ANFIS and GARIC are used in control systems [5], FALCON for parametric identification in adaptive automatic control systems (ACS) with a tunable model [5], NEFCON in synthesis tasks when dealing with a black box object [5], FUN in control tasks in mobile robotics [6], and fuzzy multilayer perceptron for the identification of data sets [4]. In [4] a class of hybrid artificial neural networks, which use fuzzy neurons with fuzzy inputs and outputs and/or fuzzy weights, but with a clear activation function, is separately identified. The peculiarity of all considered FANN is the presence of a special layer - a layer of rules, in which intermediate operations of data transformation are performed using fuzzy logic operations [4-5].

This feature complicates the development of FANN training algorithms, because in addition to calculating the value of synaptic weights of the links it is necessary to adjust the fuzzy inference rules for the layer of rules, and, possibly, the parameters of the fuzzy layer membership functions.

Thus, the number of fuzzy layer rules depends on the number of input variables and the number of their terms, and can be determined by the formula [4]:

$$
N_{r}=N_{i n}^{N_{t}},
$$

where $N_{r}$-number of rules, $N_{i n}$-number of input variables, $N_{t}$ - number of terms

Consequently, for a network with five input variables, each of which is represented by three terms, the number of such rules will be 125 . As the number of rules increases, the time spent on training of FANN and the algorithm of its training will also increase accordingly.

Some publications propose to involve experts to tune membership functions of a fuzzy layer [7], which creates certain difficulties: first, it is necessary to involve as many experts as possible for an adequate representation of knowledge, and second, each expert will contribute his subjective error. The authors of this article believe that the FANN should be adjusted without involving experts, but on the basis of the available real data.

We propose the structure of a fuzzy artificial neural network corresponding to the "classical" multilayer perceptron [3] with two hidden layers. Figure 1: shows an example of such FANN for the case of two input variables. 


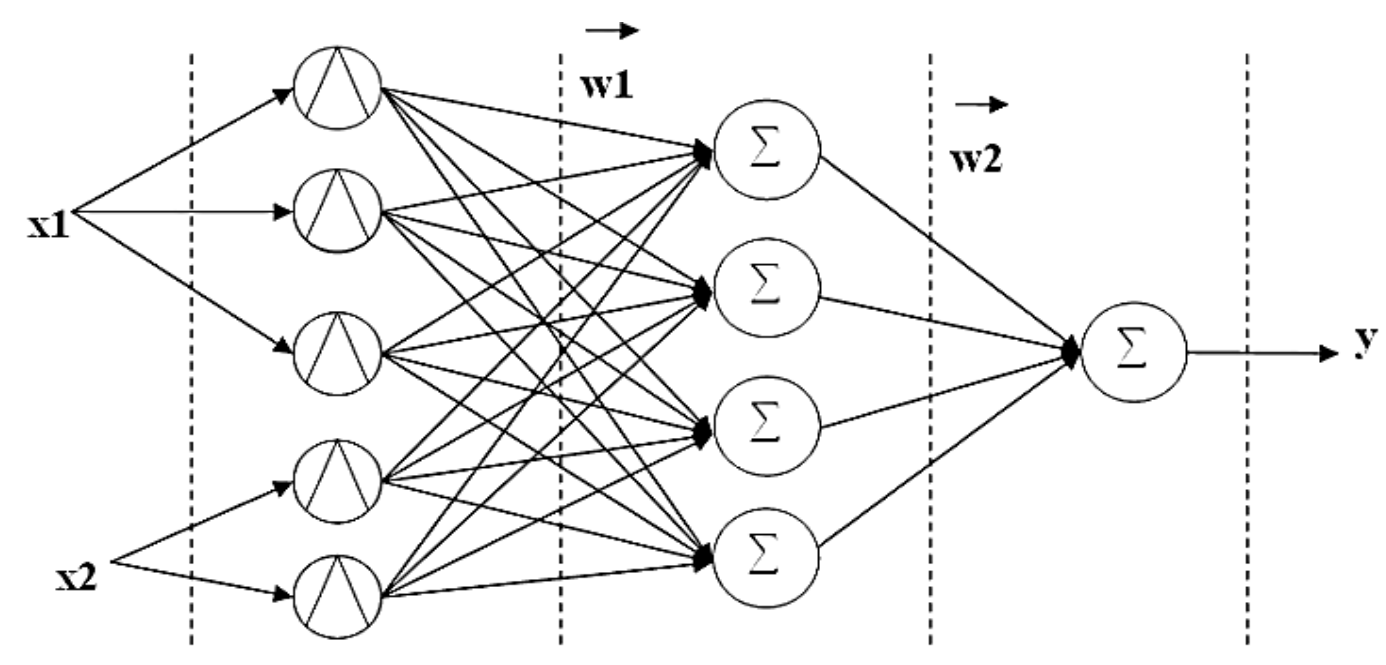

1 layer 2-layer

Figure 1: Structure of the proposed FANN

4-layer

5-layer

The proposed FANN contains five layers:

1. input data layer,

2. first hidden fuzzy layer (phasing layer - fuzzy reduction),

3. second hidden layer containing neurons with linear activation function, performing summation of data obtained in the second layer multiplied by synaptic weights vector $\mathrm{w} 1$,

4. layer performing summation of data obtained in the third layer multiplied by synaptic weights vector $\mathrm{w} 2$,

5. output data layer.

The number of neurons in the first hidden (fuzzy) layer is equal to the sum of the number of terms of all input variables [8].

The number of neurons in the second hidden layer is determined in the process of FANN adjustment. At the initial stage this index can be chosen equal to the number of neurons in the first hidden layer.

The fourth layer is resultant, so it consists of one neuron with linear activation function.

Based on the notation system accepted in literature, we can write the structure of FANN shown in Figure 1: as 2-5-4-1. To explain the working principle of the first hidden layer of the proposed FANN, consider the membership functions - the terms of the first hidden layer for one input variable. Suppose there are three such terms, as shown in Figure 2:.

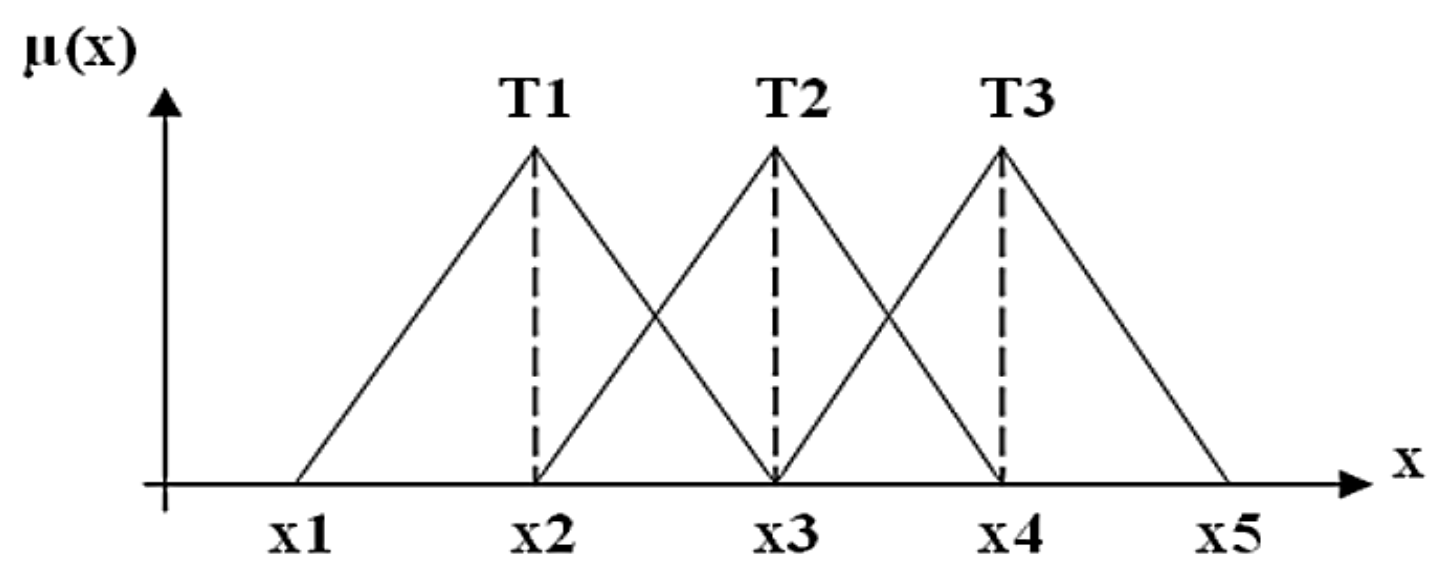

Figure 2: Terms of the first hidden FANN layer for a single input variable

Then on the interval $\mathrm{xl}-\mathrm{x} 2$ only the effect of the term $\mathrm{T} 1$ will appear, on the interval $\mathrm{x} 2-\mathrm{x} 3 \mathrm{-}$ - the terms and $\mathrm{T} 2$, on the interval $\mathrm{x} 3-\mathrm{x} 4$ - the terms $\mathrm{T} 2$ and $\mathrm{T} 3$, and on the interval $\mathrm{x} 4-\mathrm{x} 5$ - the term $\mathrm{T} 3$. This means that when you change the values of the input variable $\mathrm{x}$ in the interval $\mathrm{xl}-\mathrm{x} 5$, the values of the 
coefficients by which the elements of the vector wl are multiplied, which then arrive to the neurons of the second hidden layer, will change. The selection of membership functions for the first hidden layer of FANN can be done in two ways. The first way is to distribute the accessory function carriers uniformly in the carrier domain for the whole range of possible values of input variables, as shown in Figure 2:, and the second way is based on the processing of initial statistical information [8].

To verify the proposed structure of FANN, let's take the data of the half-hour graph of the power consumption of the coal mining enterprise for a day. Figure 3: shows a graph of the original data, and predicted values, obtained with the proposed FANN with the structure 2-5-3-1 and with the structure 2-14-5-1.

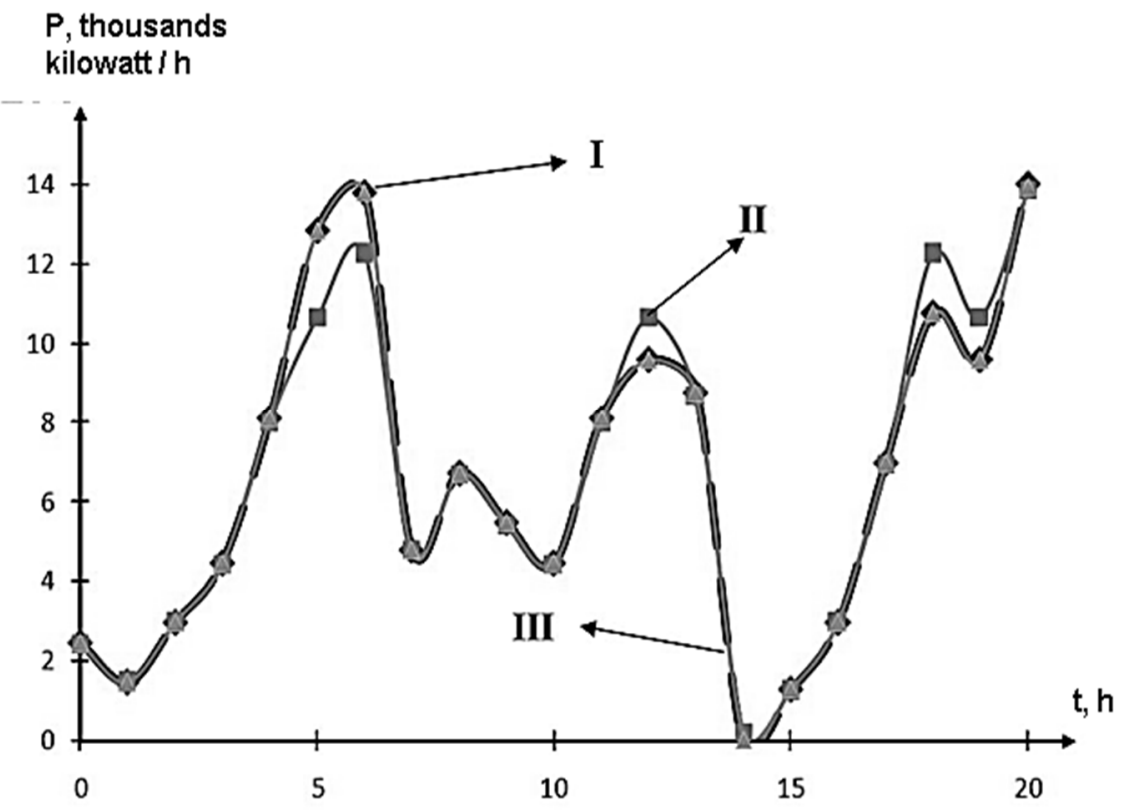

Figure 3: Graphs of initial and forecast data

I. the original time series (dotted line);

II. time series synthesized by FANN (2-5-3-1);

III. time series synthesized by FANN (2-14-5-1)

To estimate the training time and RMS errors, NINSs with different structures were constructed. Since the number of input variables is constant in all cases (two variables), the parameters of tested NINSs are represented by the number of neurons in the first hidden layer N HL1 and the second hidden layer N HL2. The results are shown in Table 1

\section{Table 1}

Differ by several orders of magnitude, the error values on the ordinate axis are plotted in the logarithmic scale

\begin{tabular}{|c|c|c|c|c|}
\hline \multirow[t]{2}{*}{ № } & \multicolumn{2}{|c|}{ Structure of FANN } & \multirow[t]{2}{*}{ Training time, $\mathrm{c}$} & \multirow{2}{*}{$\begin{array}{c}\text { RMS error } \varepsilon \\
\%\end{array}$} \\
\hline & $\mathrm{N}_{\mathrm{HL} !}$ & $\mathrm{N}_{\mathrm{HL} 2}$ & & \\
\hline 1 & 4 & 2 & 5.625 & 6.15 \\
\hline 2 & 4 & 3 & 8.156 & 5.37 \\
\hline 3 & 4 & 4 & 9.516 & 4.4 \\
\hline 4 & 5 & 3 & 9.953 & 5.86 \\
\hline 5 & 5 & 4 & 12.344 & 5.86 \\
\hline 6 & 5 & 5 & 15.344 & 5.86 \\
\hline 7 & 6 & 3 & 10.093 & 8.67 \\
\hline 8 & 6 & 4 & 13.062 & 5.92 \\
\hline
\end{tabular}




\begin{tabular}{ccccc}
9 & 6 & 5 & 16.062 & 2.40 \\
10 & 8 & 3 & 11.234 & 0.01 \\
11 & 8 & 5 & 17.562 & 0.011 \\
12 & 8 & 7 & 23.875 & 0.008 \\
13 & 10 & 3 & 13.797 & 2.25 \\
14 & 10 & 5 & 22031 & 2.26 \\
15 & 10 & 8 & 35860 & 2.26 \\
16 & 14 & 3 & 17.453 & 0.0045 \\
17 & 14 & 4 & 23.328 & 0.0055 \\
18 & 14 & 5 & 28.985 & 0.0031 \\
\hline
\end{tabular}

Figure 4:, Figure 5: show the dependences of the learning time and the mean-square error, plotted according to Table 1 . Since the minimum and maximum error values in Table 1 differ by several orders of magnitude, the error values on the ordinate axis are plotted in the logarithmic scale.

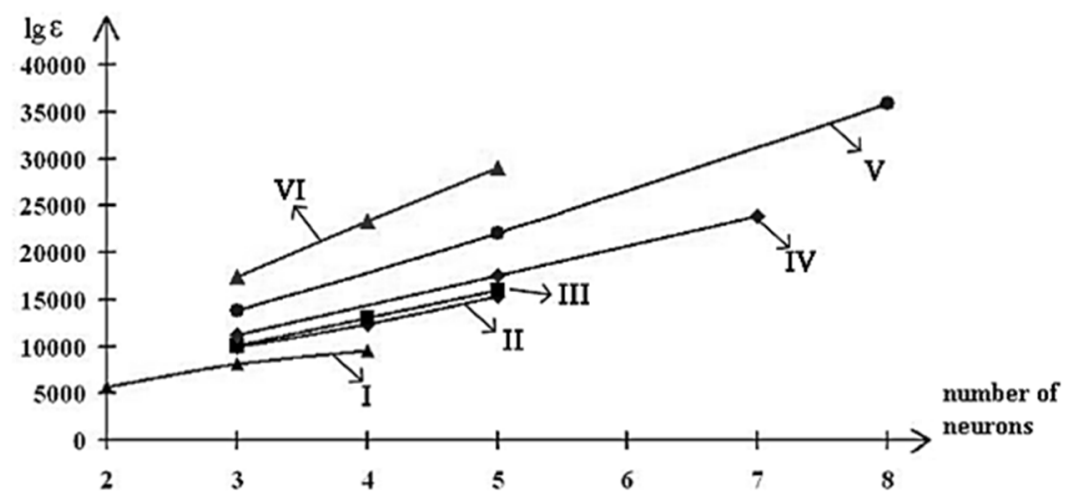

Figure 4: Plot of the root-mean-square error obtained in the FANN

as a function of the number of neurons in the second hidden layer:

I. a network with 4 IF;

II. a network with 5 IF;

III. a network with 5 IF;

IV. a network with 8 IF;

V. a network with $10 \mathrm{IF}$;

VI. a network with 14 IF

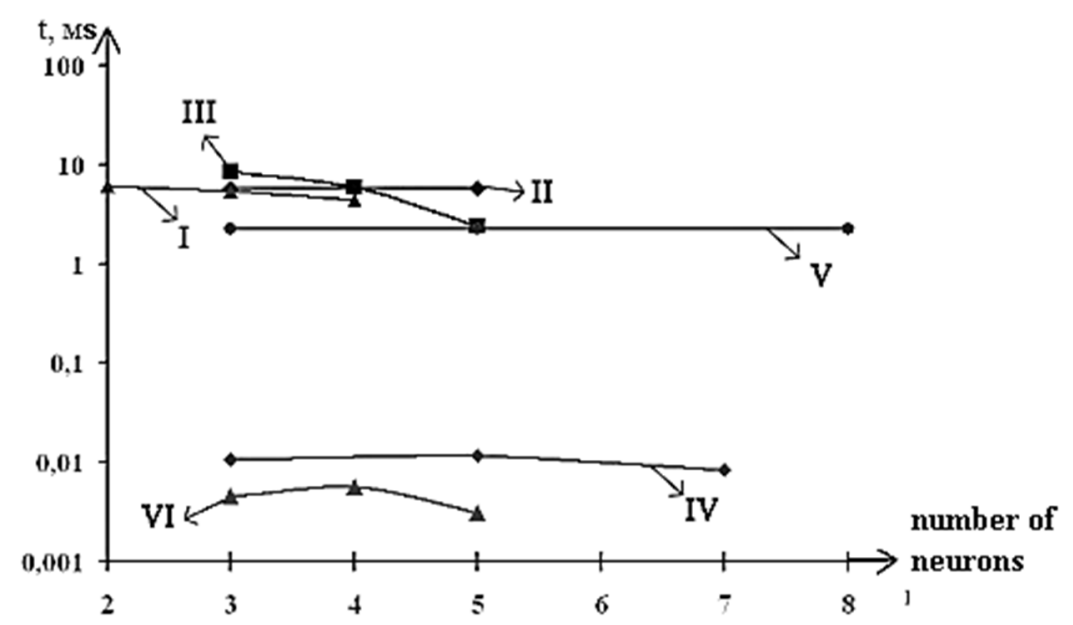

Figure 5: Graph of the dependence of the training time of the FANN 
as a function of the number of neurons in the second hidden layer:

I. network with 4 IF;

II. a network with 5 IF;

III. a network with 5 IF;

IV. a network with 8 IF;

V. a network with $10 \mathrm{IF}$;

VI. a network with 14 IF.

From the above dependencies of Figure 4:, Figure 5: The number of neurons in the first hidden layer has the greatest influence on the learning time and the error value.

The accuracy of prediction obtained with the proposed FANN can be improved by adjusting the accessory functions for hidden layers. Thus, for example, a slight modification of the parameters of membership functions for the first hidden layer, shown in Figure 6:, Figure 7: for FANN with the structure $2-5-5-1$, allows to reduce the error of $5,86 \%$ to $0,11 \%$ (version 1 ) and $0,19 \%$ (version 2 ).

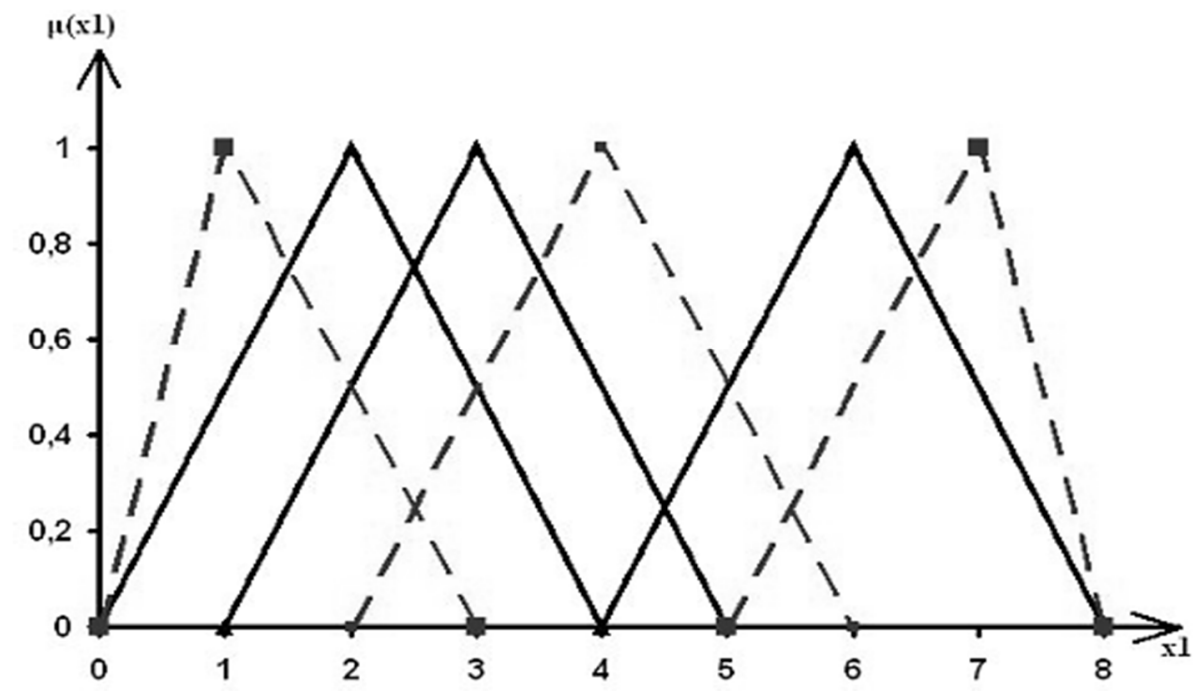

Figure 6: Identity functions for the three terms of the first variable: solid line-version 1; dotted lineversion 2

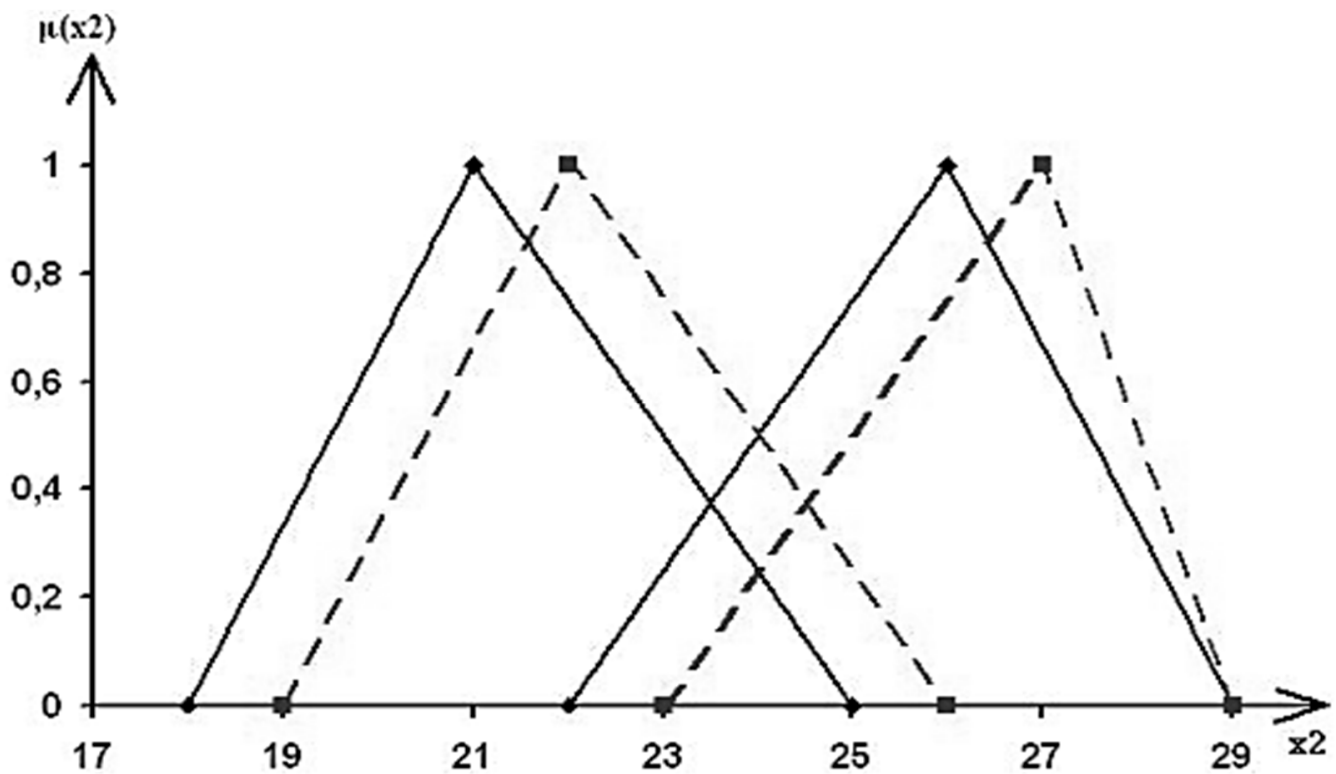

Figure 7: Identity functions for two terms of the second variable: solid line-version 1; dotted lineversion 2 


\section{Conclusions}

Exclusion of fuzzy rules layer from the structure of fuzzy artificial neural network, built on the structure of perceptron with two hidden layers, allows to simplify the procedure of its training and does not limit the researcher in the number of fuzzy neurons. Exclusion of the rules layer will also allow to refuse the subjective component introduced by experts. The presence of fuzzy in the first hidden layer provides an opportunity to train a neural network with a small amount of input data.

\section{References}

[1] S. Sulzberger, FUN: Optimization of Fuzzy Rule Based Systems Using Neural Networks / S. Sulzberger, N. Tschichold-Gtirman, S. Vestli, IEEE International Conference on Neural Networks (ICNN-93), San-Francisco, California, volume 1, pp. 312-316, 1993.

[2] Ye. I. Kucherenko, V. A. Filatov, I. S. Tvoroshenko, R. N. Baidan, Intellectual technologies in decision-making technological complexes based on fuzzy interval logic, East European Journal of Advanced Technologies 2 (2005) 92-96.

[3] Coskun-Setirek, Z. Tanrikulu, Intelligent interactive voice response systems and customer satisfaction, International Journal of Advanced Trends in Computer Science and Engineering 8(1) (2019) 4-11. https://doi.org/10.30534/ijatcse/2019/02812019.

[4] D. Fontes, P. A. Pereira, F. Fontes, A Decision Support System for TV self-promotion Scheduling, International Journal of Advanced Trends in Computer Science and Engineering 8(2) (2019) 134140. https://doi.org/10.30534/ijatcse/2019/06822019.

[5] S. Tvoroshenko, Structure and functions of intelligent decision-making tools in complex systems, Artificial Intelligence 4 (2004) 462-470.

[6] Ye. I. Kucherenko, I. S. Tvoroshenko, Operative evaluation of the space of states of complex distributed objects using fuzzy interval logic, Artificial Intelligence 3 (2011) 382-387.

[7] S. Tvoroshenko, Analysis of Decision-Making Processes, Intelligent Systems, Information Processing Systems 2 (2010) 248-253.

[8] S. Egorov, A. N. Shaykin, Logical modeling under uncertainty based on fuzzy interval Petri nets, News of the Russian Academy of Sciences, Theory and Control Systems 2 (2002) 134-139. 